\title{
The Assessment of Forced Penetration: A Necessary and Further Step Toward Understanding Men's Sexual Victimization and Women's Perpetration
}

\author{
RaeAnn E. Anderson \\ University of North Dakota, raeann.anderson@UND.edu \\ Erica L. Goodman \\ Sidney S. Thimm
}

\section{How does access to this work benefit you? Let us know!}

Follow this and additional works at: https://commons.und.edu/psych-fac

Part of the Criminology and Criminal Justice Commons, and the Psychology Commons

\section{Recommended Citation}

RaeAnn E. Anderson, Erica L. Goodman, and Sidney S. Thimm. "The Assessment of Forced Penetration: A Necessary and Further Step Toward Understanding Men's Sexual Victimization and Women's Perpetration" (2020). Psychology Faculty Publications. 32.

https://commons.und.edu/psych-fac/32 


\title{
The Assessment of Forced
} Penetration: A Necessary and Further Step Toward Understanding Men's Sexual Victimization and Women's Perpetration

Journal of Contemporary Criminal Justice

(C) The Author(s) 2020

Not the version of record. The version of record is available at https://doi.org/10.1 I 77/2FI043986220 $\underline{936108}$

journals.sagepub.com/home/ccj

\section{RaeAnn E. Anderson' $₫$, Erica L. Goodman ${ }^{1,3}$, and Sidney S. Thimm'}

\begin{abstract}
A unique form of sexual victimization that often goes undiscussed and, therefore, underassessed is that of being forced to penetrate another person (i.e., forced penetration). Due to forced penetration being a relatively novel addition to the definition of rape, there is a lack of assessment tools that identify forced penetration cases. Thus, the goal of this study was to assess the utility and validity of new items designed to assess forced penetration. More than I,000 participants were recruited across three different studies to assess forced penetration victimization and perpetration. The rate of forced penetration victimization ranged from $4.5 \mathrm{I} \%$ to $10.62 \%$. Among men who reported victimization of any type, $33.8 \%$ to $58.7 \%$ of victimized men reported experiencing forced penetration across the samples, suggesting this experience is common. All new and unique cases of sexual victimization identified by the forced penetration items were those of heterosexual men. These findings suggest that assessing for forced penetration would increase the reported prevalence rates of sexual victimization, particularly in heterosexual men (and correspondingly, rates of perpetration in women).
\end{abstract}

\section{Keywords}

forced penetration, sexual victimization, sexual perpetration, men, measurement, assessment, female perpetration

I Department of Psychology, University of North Dakota, Grand Forks, ND, USA

2 Department of Psychological Sciences, Kent State University, Kent, OH, USA

3 Department of Psychiatry \& Behavioral Sciences, Northwestern University, Evanston, IL, USA

\section{Corresponding Author:}

RaeAnn E. Anderson, University of North Dakota, Department of Psychology, 2000 Columbia Hall, Grand Forks, ND 58202, USA. Email: raeann.anderson@UND.edu. 
Although most scholarship and clinical work focuses on the sexual victimization experiences of young women due to the high prevalence of this crime, recent data suggests approximately one in five men in the United States experience sexual violence victimization as well (Black et al., 20II). However, the research on sexual perpetration overwhelmingly focuses on men as perpetrators (Fisher \& Pina, 20I3); until recently definitions of rape frequently outright excluded the experiences of men being harmed and women harming them (Stemple \& Meyer, 20l4). We define sexual violence as any form of sexual contact without consent (Basile, Smith, Breiding, Black, \& Mahendra, 20I4). In this study we will use the term sexual victimization to refer to the experiences of those who have been harmed by sexual violence and the term sexual perpetration to refer to the behavior of those who harm others through sexual violence, irrespective of gender identity. Rape, the most severe form of sexual violence, is oral, anal, or vaginal penetration by means of substance use intoxication, threats of physical force, or use of force (Basile et al., 20I4; Koss et al., 2007). The consequences of sexual victimization can be profoundly serious. Rape is associated with high rates of posttraumatic stress disorder and poorer health (Dworkin, Menon, Bystrynski, \& Allen, 2017; Koss, 1993), regardless of a person's gender (Choudhary, Coben, \& Bossarte, 2009; Weiss, 2010). The purpose of this study was to evaluate the usefulness (i.e., utility) and validity of three new items designed to assess forced penetration, a form of sexual violence more often experienced by men, and thus propel research on this topic.

\section{Prevalence and Characteristics of Sexual Victimization in Men}

Forced penetration is when someone is forced to use parts of their body to penetrate another person's body. Until recently in the last decade, most legal definitions of rape excluded forced penetration and many research tools have also excluded this experience (Stemple \& Meyer, 20I4). Not only does this strategy under-estimate and discount men's experiences of victimization, this also systematically obscures women's perpetration. Yet, research suggests that this experience is traumatizing for men and may be an under-recognized form of violence against heterosexual men by heterosexual women (Brousseau, Bergeron, Hébert, \& McDuff, 20I I; Fisher \& Pina, 20I3; Weare, 2018b; Weiss, 20I0). However, it is notable that though initial research suggests forced penetration victimization may be more common for heterosexual men, forced penetration can occur in any circumstance and with many combinations of people with varying gender and sexual identities.

The Centers for Disease Control (CDC) data suggests that approximately $22 \%$ of American men experience sexual victimization. Data from college men indicate higher rates $-28-50 \%$ (Anderson, Cahill, \& Delahanty, 20I8a; Turchik, 20I2) with reported rates of rape being slightly lower than those reported by college women. In any case, the rate of sexual victimization in men is much higher than previously purported, consistent with Stemple \& Meyer's (2014) suggestion. In light of old assumptions about men being much less likely to be victimized sexually, research is just beginning to examine how men's experiences may differ than women's (Weiss, 2010).

For instance, the gender of those perpetrating against men is unclear. Data from adolescents suggest perpetrators against men are almost exclusively women (95\%: French, Tilghman, \& Malebranche, 2015) as does some data with college men and community men (62-73\%: Anderson et al., 2018a; Wegner \& Davis, 2017) and review papers (Davis, 2002). In contrast, data from the US National Crime Victimization Survey suggest the perpetrators are mostly men - 65\% (as cited in Stemple \& Meyer, 20 I4). In research with heterosexual couples, $30 \%$ reported male sexual victimization, and by design all perpetrators were women (Brousseau et al., 20l I). This conflicting data is likely partially a result of some studies including forced penetration (e.g., Wegner \& Davis, 2017) whereas other studies do not. Beyond CDC data, very few studies report the prevalence of forced penetration or whether forced penetration identifies cases of victimization that would have otherwise not recognized and thus, the harm of this offense unaddressed.

\section{Forced Penetration \& Measurement Issues}

One reason for the opacity regarding the reality of men's sexual victimization is the highly variable measurement strategies that have been used to investigate this issue (Peterson, Voller, Polusny, \& Murdoch, 20 I0). Indeed, as eloquently summarized by Stemple \& Meyer (20 I4), until 20 I2, the Federal Bureau of Investigation in the United States and many other governmental agencies used a restrictive and gender biased definition of rape, dictating that rape requires the victim to be female and physically forced. These sexist assumptions have not been unique to the United States; the United Kingdom defines sexual violence 
by men's behavior and classifies cases where men are penetrated in a less serious legal category (Weare, 2018a).

The research literature has been less gender biased and restrictive, yet still infrequently explores men's victimization. For example, even though the original Sexual Experiences Survey (SES) was revised to reduce gender bias (producing the Sexual Experiences Survey - Short Form Victimization), the SES-SFV does not assess forced penetration of a vagina by a penis. Further, research suggests that the emphasis on sexual behaviors and descriptions of genitalia in the SES-SFV inherently genders the questionnaire which may introduce bias that decreases men's reporting (Anderson, Cahill, \& Delahanty, 2018b; Anderson \& Delahanty, 2019). Multiple studies have demonstrated that questionnaires which are less specific about sex and genitalia identify more cases of male sexual victimization (Anderson, Cahill, \& Delahanty, $2018 b ;$ Anderson \& Delahanty, 2019; Struckman-Johnson, Struckman-Johnson, \& Anderson, 2003). However, the weakness of questionnaires that are less specific about the sexual acts is that although phrases like "forced you to have sex" may encompass forced penetration but one would be unable to identify specific cases and therefore examine the prevalence rates or predictors of forced penetration.

As of this writing, we are unaware of any standardized questionnaires that assess forced penetration specifically. Although gender-neutral questionnaires such as the, Revised Conflict Tactics Scales and the Post-Refusal Persistence Scale-Victimization document higher rates of sexual victimization for men, the lack of precise information on the sexual behaviors coerced obfuscates cases of forced penetration. More specific attempts, such as the CDC's National Intimate Partner and Sexual Violence Survey (NISVS) do retain information on specific sexual behaviors and tactics but, to our knowledge, have not published data on the psychometric properties of this questionnaire.

Finally, some argue that even gender neutral questionnaires which have been designed based on the existing literature may contain gender bias because the current literature largely studies the common and stereotypical male perpetrator/female victim dyad and therefore may not reflect the potentially unique tactics and strategies of women who perpetrate (Buday \& Peterson, 20I5). Indeed, one study demonstrated a false positive rate of nearly $40 \%$ on the SES-Long Form Perpetration for women, suggesting that the measurement of sexual perpetration by women may be challenging (Buday \& Peterson, 2015). Although there have been several studies to establish the construct validity of Sexual Experiences Surveys to assess perpetration by men (Koss \& Gidycz, 1985; Ross \& Allgeier, 1996; Ouimette, Shaw, Drozd, \& Leader, 2000) to our knowledge only one has examined this with women (Buday \& Peterson, 20I5).

\section{The Current Study}

The goal of the current study is to improve the understanding of men's experiences of sexual victimization by designing and testing new methods to assess forced penetration. We designed new items in the style of the SES-SFV, in order to obtain specific information on the sexual acts while still assessing a range of tactics, and assessed their utility and validity in three samples: two samples of college students and one sample of workers from Amazon Mechanical Turk (MTurk workers: individuals that receive payment for completing research studies crowdsourced through Amazon.com). Our study is innovative in that we test victimization items as well as perpetration items and we tested these items with persons of all genders. Although the CDC NISVS data suggest that forced penetration victimization against women is extraordinarily rare (Black et al., 20I I) we chose to administer these items to all participants in the spirit of inclusion and equity. Administering forced penetration items to all participants also provides another empirical test of gender differences in forced penetration as it is possible that a women could forcibly penetrate another person using an object or fingers. We assess the utility of these items by examining how many unique cases of sexual victimization are identified and assessing missing data as a proxy for participant comprehension of these items. We evaluate validity by examining correlations with other SESSFV items. Finally, we examine the characteristics of cases of forced penetration (gender of the perpetrator, relationship to the perpetrator) hypothesized to be characteristic of forced penetration. Specifically our hypotheses $(\mathrm{H}) /$ research questions $(\mathrm{RQ})$ were:

$R Q$ I: Document the rate of forced penetration victimization in a high-risk sample (college students). We will also investigate whether assessing forced penetration results in higher prevalence rates by expanding the scope of cases identified.

$R Q$ 2: Evaluate which type of forced penetration (completed vaginal, completed anal, attempted anal) is most common.

$R Q$ 3: Analyze patterns of missing data as a proxy for participant comprehension. 
HI: We hypothesize that we will find good evidence of validity for the items assessing forced penetration victimization $(\mathrm{HIa})$ and perpetration $(\mathrm{HIb})$ as demonstrated by correlations with other SES-SFV items ( $r>.4$, Anderson, Cahill, Delahanty, 2018a) and SES-SFP items ( $r>.5$, Anderson, Cahill, Delahanty, 2017).

$H 2$ : We hypothesize that the perpetrators of forced penetration will be more likely to be women $(\mathrm{H} 2 \mathrm{a}$, consistent with Weare, $20 \mathrm{I} 8 \mathrm{a})$ and someone known to the victim $(\mathrm{H} 2 \mathrm{~b})$, especially compared to those identified as rape victims by the traditional SES-SFV items.

All RQs/Hs were carried out in Study I using two samples of college students using forced penetration victimization items. We also explored the same $\mathrm{RQs}$ and $\mathrm{HI}$ regarding forced penetration perpetration in a sample of MTurk workers.

\section{Method}

\section{Participants}

Study I, Sample I. Participants were 673 college students at a large, public, Midwestern university with a mean age of $19.5(S D=3.6)$. Women comprised $54.5 \%(n=367)$ of the sample, men - $44.3 \%(n=298)$, and transgender and other gender identities - I. $1 \%(\mathrm{n}=8$ gender minorities). Most participants reported being heterosexual (85.6\%); some were bisexual (5.6\%), gay (4.9\%), and queer (1.5\%). A small number of participants reported their sexual orientation as other $(n=31$; $14.4 \%$ of the 31 represent sexual minorities); on reviewing the descriptive labels provided by participants a minority of these participants were re-classified as heterosexual because they provided self-labels consistent with heterosexuality (e.g., "regular" or "straight"). Participants were mostly Caucasian (85.6\%); some were African American (9.4\%), Asian American (3.7\%), and Native American (1.0\%). A few participants reported identifying as Latinx (3.7\%). Another study utilizing this sample has been published (Anderson \& Delahanty, 2019); this study focused on comparing two different sexual victimization questionnaires to improve measurement strategies. This prior study included the forced penetration items to calculate prevalence rates but did not further analyze these items.

Study I, Sample 2. Participants were 792 college students at a large, public, Midwestern university. Nine participants were excluded from analyses due to missing or implausible age (e.g., 99 years); final number of participants used in analyses was 783 with a mean age of $19.6(S D=1.71)$. Women comprised $72.3 \%$ $(n=566)$ of the sample, men $-26.3 \%(n=206)$, and genderqueer and other gender identities $-1.4 \%(n=$ I I gender minorities). Most participants reported being heterosexual (83.0\%); some were bisexual (8.4\%), gay (3.5\%), and queer (.6\%; in total sexual minorities account for $15.2 \%$ of the sample). A small number of participants reported their sexual orientation as other $(n=30)$; similar Sample I a small number of these participants were recoded as heterosexual when they wrote in responses such as "straight". Participants were mostly Caucasian (8I.6\%); some were African American (13.2\%), Asian American (2.9\%), and Native American (.8\%). A few participants reported identifying as Latinx (4.9\%).

Study 2, Sample 3. Participants were 293 American MTurk workers with a mean age of 32.16 (SD = 8.38). Women comprised $53.2 \%(n=156)$ of the sample, men $-45.1 \%(n=132)$, and other gender identities - $1.7 \%$ ( $n=5$ gender minorities). Most participants reported being heterosexual (82.9\%); some were bisexual (I I.6\%), gay (3.8\%), and other (I.7\%; a total of $17.1 \%$ represent sexual minorities). A small number of participants reported their sexual orientation as other $(n=30)$; none of these participants provided a further written response. Participants were mostly Caucasian (81.6\%); some were African American (13.2\%), Asian American (2.9\%), and Native American (.8\%). Some reported identifying as Latinx (4.9\%).

\section{Measures}

The Sexual Experiences Survey - Short Form Victimization (SES-SFV: Koss et al., 2007). All SES-SFV items are behaviorally-specific items that begin with a stem describing a sexual behavior (i.e., "A man put his penis into my vagina, or someone inserted fingers or objects...") followed by five possible tactics listed below that were used by the perpetrator to coerce the survey respondent. These tactics can be grouped in the five following categories: verbal criticism, verbal pressure, alcohol incapacitation, threats of physical force, and physical force. This generates 35 items for women ( 7 sexual behaviors $X 5$ tactics) and 25 items for men ( 5 sexual behaviors $X 5$ tactics). The traditional instructions starting with, "The following questions concern sexual experiences that you may have had that were unwanted. We know that these are personal questions, so we do not ask your name or other identifying information..." were given. Items are 
ordered roughly hierarchically starting with less severe items; in Sample I the items were given in the order set by Koss et al., (2007), in Sample 2 items were given in a randomized order. The SES-SFV has shown good evidence of convergent validity and test-retest reliability in past research with college-aged women (Johnson, Murphy, \& Gidycz, 20 I7). It has also demonstrated good evidence of convergent validity with college-aged men and adequate evidence of test-retest reliability when scored dichotomously (Anderson et al., 2018a). In Study I, we utilized a dichotomous scoring to assess whether each experience (e.g., item) had ever taken place since age 14. After completing the SES-SFV items participants were asked questions to ascertain the gender and relationship to the perpetrator.

Forced penetration items (victimization). Given our interest in gender differences in the current study, we modified the SES-SFV to be more gender neutral and inclusive following Hipp \& Cook (2017)'s suggestions for research and evidence from Anthony \& Cook (20I2). For example, item 3 on the SES-SFV assesses completed vaginal penetration but only for women. However, it is possible for a male to be forced to penetrate a woman's vagina with his penis or a woman to be forced to penetrate a vagina with fingers or objects; thus, we added three items designed to more broadly capture possible experiences of being forced to engage in vaginal or anal sex. For example, "A woman MADE ME put my penis into her vagina, or MADE ME insert my fingers or objects into her vagina without my consent" assesses completed forced penetration of a vagina for both men and women. Similarly, "Someone MADE ME put my penis into their butt, or someone MADE ME insert my fingers or objects without my consent" assesses forced penetration of the anus. Finally, "Even though it didn't happen, a man tried to MAKE ME put his penis into my butt, or someone tried to MAKE ME stick in objects or fingers without my consent" assess attempted anal forced penetration. These three additional items were added for all genders such that our revised version of the SES-SFV contained 50 items for women (I 0 sexual behaviors $\times 5$ tactics) and 40 items for men ( 8 sexual behaviors $\times 5$ tactics). All participants were presented with all items; however, an additional instruction preceded the vaginal penetration items, "skip this item if you do not have a vagina" to direct participants with penises to the remaining items.

The Sexual Experiences Survey - Short Form Perpetration (SES-SFP: Koss et al., 2007). The SES-SFP is written very similarly to the SES-SFV and assesses the same sexual behaviors and tactics since age I4. Age I4 was chosen by the original authors to contrast from childhood sexual abuse. The traditional SES-SFP contains 35 items (7 sexual behaviors X 5 tactics). The SES-SFP has demonstrated strong evidence of validity and good evidence of test-retest reliability with college men in past research (Johnson et al., 2017; Anderson, Cahill, \& Delahanty, 2017). To emphasize that the SES-SFP was not assessing victimization (as has been a problem in other research, see Buday \& Peterson, 2015) the following text was added as instructions before the questionnaire, "These are questions about things you have done to someone else since the age of 14." One additional tactic of perpetration was included in this study; multiple perpetrator attacks were assessed for every sexual behavior with the language, "Acting as part of a group of two or more people who did these things together," the wording for this item was modeled after the SES-Long Form Perpetration (Koss et al., 2007).

Forced penetration items (perpetration). Four items were added to assess four types of forced penetration, attempted vaginal, completed vaginal, attempted anal, and completed anal. Similar to the added forced penetration victimization items described above, these items were written in the same style as the traditional SES-SFP items (behaviorally, specific, compound) but nouns and verbs were changed to specify perpetration. Thus SES-SFP in this study contained a total of 66 items (I I sexual behaviors X 6 tactics). In Study 2, Sample 3, we utilized Qualtrics display logic to only administer the vaginal penetration items to participants with vaginas.

Differences between the victimization and perpetration questionnaires. The forced penetration items were added to both victimization and perpetration questionnaires and all questionnaires were scored dichotomously. However, the SES-SFP used in this study contained more items due to the additional of attempted vaginal forced penetration items (which were inadvertently left out of Study I) and the addition of multiple perpetrator tactics.

Scoring. All SES-SFV and SES-SFP items were scored dichotomously, any response of I or greater was considered affirmative for that item. Composite scores were created for the SES-SFV and SES-SFP traditional and added forced penetration items given that each item is a type of sexual behavior with 5 subitems that identifies the tactic used. The sub-items were totaled to give a composite score for each type of sexual behavior when comparing to other items.

Most measures used in social science research are based on a latent measurement model as most social science constructs cannot be directly observed, e.g., seen and measured. In contrast, the measurement of sexual violence is the assessment of a history of behaviors or experiences. Each experience or behavior is not necessarily related and may have occurred in different incidents, perpetrated by different 
people, et cetera. Thus, assuming a latent measurement model for sexual violence history is unwarranted and atheoretical as individual experiences of sexual victimization are not presumed to be caused by some underlying latent factor (Koss et al., 2007) and reporting Cronbach's alpha is designed for latent models (Diamantopoulos, Riefler, \& Roth, 2008;Koss et al., 2007).

\section{Procedures}

The SES-SFV was administered in Samples I and 2 while the SES-SFP was administered in Sample 3. In Sample I participants completed the study questionnaires in a set order, in Samples 2 and 3 participants completed all questionnaires in a randomized order. Samples I and 3 contained only sexual violence history questionnaires and demographics. Sample 2 included several other questionnaires pertaining to the broader goals of that study (the effect of how a study was advertised on victimization and perpetration outcomes) including constructs such as alcohol, health, attitudes related to rape, and personality which are not presented here. Data for Study I were collected from September - December 2017; data for Study 2 were collected from February - May 2018; data for Study 3 were collected in May 2017.

\section{Data Analytic Plan}

Participants who completed less than $20 \%$ of the study were summarily excluded from the datasets. In addition, participants who provided implausible data (e.g., age of 99) were also excluded. Data that were otherwise missing were assumed to be missing at random and for the sexual violence questionnaires replaced with the modal value (0), except for the forced penetration items. We analyzed the rate of missing data among the forced penetration items separately given their unknown psychometric properties. Specifically, we were concerned that participants who didn't understand the forced penetration items may just skip them; thus, replacing the forced penetration items with the modal value might incorrectly conflate misunderstanding with lack of endorsement. In analyzing the relationship to the perpetrator we do not present data on relative perpetrators due to the low number of cases compared to other types of perpetrators $(4.5-9.1 \%)$.

\section{Results}

\section{Descriptive Results}

In Sample I, $21.8 \%$ of men and 51.5\% of women reporting sexual victimization of any type on the SESSFV; in Sample 2, 22.3\% of men and 48. I\% of women reported sexual victimization of any type. In Sample $3,12.1 \%$ of men $(n=16)$ and $10.3 \%$ of women $(n=16)$ reported sexual perpetration of any type.

\section{Victimization}

$R Q$ I: Prevalence of forced penetration victimization. Rates of experiencing forced penetration (all types) in college samples were $4.5 \mathrm{I} \%(n=30$, Sample I) and I0.62\% $(n=82$, Sample 2$)$, see Table I for further details described by type of forced penetration and gender of participant. In Sample 1, 7.4\% of the entire sample of men and $33.8 \%$ of victimized men experienced forced penetration $(n=22)$. In Sample 2, $13.1 \%$ of the entire sample of men and $58.7 \%$ of victimized men experienced forced penetration. Men $(67.5 \%)$ were significantly more likely to report experiencing forced penetration victimization than women in Sample 1 $\left(32.93 \%\right.$; $\left.X^{2}(I, 665)=10.31, P<.01\right)$, but not in Sample $2\left(13.1 \%\right.$ vs. 9.7\%: $X^{2}(I, 773)=1.84$, $p=.175)$. There were no differences in the rate of forced penetration experiences reported between heterosexual and sexual minority men in either sample.

Unique Cases: Responses to the SES-SFV (without forced penetration questions) were compared to the forced penetration items in order to see if the addition of these items identified new cases of sexual victimization that were missed by administration of the traditional SES-SFV items alone. In Sample I for men, 6 unique cases were identified that endorsed zero SES-SFV items but did endorse forced penetration; adding in and accounting for these unique cases ( 59 cases identified by the traditional SES-SFV + 6 forced penetration cases), $9.2 \%$ of all men's sexual victimization cases were uniquely accounted for by the forced penetration items (6/65). All 6 cases were heterosexual men. No unique cases were identified for women although 8 women did endorse forced penetration.

In Sample 2, 7 unique cases of forced penetration were identified (6 heterosexual men, I heterosexual women) where participants would not have been identified by the existing SES-SFV items. Considering 
men, adding in and accounting for these unique cases ( 40 cases identified by the traditional SES-SFV +6 forced penetration cases), 13.04\% of all men who reported some type of sexual victimization were uniquely identified by the forced penetration items (6/46), most of them heterosexual men. Twice as many women, $n=55$ also reported some type of forced penetration, only I of these represented a unique case.

RQ2: Types of Forced Penetration. In Sample I, considering all participants, completed vaginal was the most frequent type of forced penetration reported $(n=19)$, followed by attempted anal $(n=10)$ and completed anal $(n=4)$ forced penetration. In Sample 2, attempted anal was the most prevalent $(n=60)$, followed by completed anal $(n=24)$, and completed vaginal $(n=29)$ forced penetration were reported at similar rates. These patterns differed slightly when only men were analyzed, see Table I. Overall, completed vaginal was the most common type of forced penetration reported by college men whereas attempted anal was the most common type reported by college women.

$R Q$ 3: Missing Data patterns: Overall, the average rates of missing responses across the composites for the three types of forced penetration types (vaginal, anal, attempted anal) were numerically higher in Sample I [vaginal (women only): 33.80\% \%, anal: 18.99\%, attempted anal: 8. I 4\%] than in Sample 2 [vaginal (women only): $13.10 \%$, anal: $9.61 \%$, attempted anal: $6.18 \%$ )]. Women were missing significantly more responses for completed vaginal and completed anal than men in Sample I $\left(X^{2}>91.95, p<.00 I\right)$, but not in Sample 2. Men had more missing responses than women for attempted anal in both samples $\left(X^{2}>\right.$ 15.95, $\mathrm{p}<.00 \mathrm{I}$ ). In comparison to the traditional SES-SFV items [vaginal penetration (women only), anal penetration, and attempted anal penetration], the traditional items had numerically less missing responses than the forced penetration items in Sample I: $1.6 \%$ (women only), 5.2\%, 6.8\%, respectively; the rates of missing data for the vaginal and anal items were also statistically different in Study I $\left(X^{2}>\mid 30.43, p<\right.$ $.00 \mathrm{I})$. The same pattern was found for Study 2 (3.2\% (women only), 5.3\%, 6.8\%, respectively), the rates of missing data for vaginal and anal penetration were statistically lower in Study $2\left(X^{2}>10.49, p \leq .00 I\right)$.

H I a: Validity. In both Samples, all three forced penetration composites (vaginal, anal, attempted anal) were significantly correlated with one another (see Table 2); these correlations were of weak to moderate strength and were stronger in Sample 2. When comparing to the traditional SES-SFV penetration items, almost all of the traditional composite items were significantly correlated with each of the three forced penetration composites in both studies, $r=.118-.663$. The only exception was the traditional SES-SFV item assessing attempted vaginal rape. We also completed these analyses by gender, results were similar.

H2: Characteristics of Victims of Forced Penetration. Gender of Participant and Relationship to the Perpetrator. In Sample I, 30 participants reported ever experiencing vaginal, anal, or attempted anal forced penetration $(73.33 \%$ male). Of these 22 males, the vast majority were heterosexual (90.91\%). Table 3 displays the data for gender of victim and gender of and relationship to the perpetrator. Overall, in Sample I men who experienced forced penetration reported significantly higher rates of female perpetration than those who experienced other forms of sexual victimization $\left(X^{2}(I, I 75)=29.624, p<.00 I\right)$. They also reported significantly higher rates of perpetration by a romantic partner $\left(X^{2}(I, 175)=10.199, p=.001\right)$. In Sample 2 , women victims of forced penetration were more likely to report female perpetrators $\left(X^{2}(I, N=255)\right.$ $=4.6 \mathrm{I}, \mathrm{p}=.032$ ) but there was no difference in the gender of the perpetrator for men. However, similarly to Sample I, men who experienced forced penetration were more likely to report romantic partner perpetrators $\left(X^{2}(I, N=255)=23.82, P<.00 I\right)$ compared to other men who reported sexual victimization.

\section{Perpetration}

RQ I: Prevalence. The rate of perpetrators endorsing forced penetration in Study 2 was 3.8\% ( $n=$ II). Of the II, 10 identified as female ( 8 heterosexual, 2 sexual minority) and I as genderqueer. Most (72.72\%) reported completed vaginal, $45.45 \%$ completed anal, $81.81 \%$ attempted vaginal, and $45.45 \%$ attempted anal forced penetration.

Unique cases. Responses to the SES-SFP (without forced penetration questions) were compared to the forced penetration items in order to see if the addition of these items identified new cases of sexual victimization that were missed by administering only the traditional SES-SFP items. One unique case was identified by the forced penetration composite that was missed by the SES-SFP; this case was a heterosexual female. In total, 33.33\% of those who endorsed perpetration of any type also reported forced penetration.

Missing data. About $70 \%$ of responses were missing for completed and attempted vaginal forced penetration; about $50 \%$ of responses were missing for completed and attempted anal forced penetration. About $40 \%$ of heterosexual and $60 \%$ sexual minority females were missing responses across the items 
for the four types of forced penetration; sexual minority women had significantly higher rates of missing data $\left(X^{2}(I, 156)=4.192, p=.04 I\right)$. Approximately half $(57.89 \%)$ of heterosexual and $46.15 \%$ of sexual minority males were missing responses on the attempted and completed forced penetration items; these rates were not significantly different $\left(X^{2}(I, I 32)=.663, p=.415\right)$. In comparison to the traditional SESSFP items the traditional items had numerically less missing responses than the forced penetration items for completed (48.1\%) and attempted vaginal (49.4\%); the rates of completed $(51.9 \%)$ and attempted anal $(5 \mathrm{I} .5 \%)$ were similar to the forced penetration questions. The rates of missing data for heterosexual women (about $40 \%$ ) and sexual minority women (about 60\%) were very similar to the forced penetration questions. This pattern was true for heterosexual men (about $60 \%$ ) and sexual minority men (46. I5\%) as well.

Validity. All 4 of the forced penetration composites were significantly correlated with one another, $r=.798-.958$; all $p<.01$ and with traditional SES-SFP composite items, $r=.50-.93 ; p<.0 \mathrm{I}$, see Table 4.

\section{Discussion}

Even though approximately one in six men experience sexual victimization (Black et al., 20I I; Anderson et al., 2018a), little is known about this phenomena. Research suggests that men may be more likely to experience forced penetration, a type of sexual victimization when someone is forced to penetrate another person's body. This study investigated the utility of new items designed to assess forced penetration in order to facilitate further research.

\section{Prevalence and Unique Cases (Utility)}

A significant number, $(7.4-13.1 \%)$ of college men reported experiencing forced penetration in this study (RQI), higher than previous research. In the NISVS study, almost 5\% of men reported forced penetration. The most common form of forced penetration victimization reported by men was vaginal, whereas this was attempted anal for women. In these studies, forced penetration accounted for 33.8 $58.7 \%$ of cases of men's sexual victimization. Additionally, 9.2-13.04\% of men's cases of sexual victimization were uniquely accounted for by forced penetration, i.e., they would have been labeled as unaffected had we not assessed forced penetration, suggesting that not measuring this type of victimization may dramatically underestimate the extent of men's sexual victimization. Notably, given the prevalence of vaginal forced penetration in our samples, this also suggests women's perpetration is being vastly underestimated.

For comparison as to whether adding forced penetration items added unique cases, Canan, Jozkowski, Wiersma-Mosle, Bradley, \& Blunt-Vinti, (2019) found two new tactics accounted for 0.9 to $2.0 \%$ of the victimization cases being newly identified. Thus, forced penetration accounts for many more unique cases than other tactics in prior literature. This was entirely specific to heterosexual men, all of the unique cases identified were of heterosexual men; further, in Study I, half of the victims of forced penetration where men, the most common form of forced penetration was vaginal and in Sample 3, none of the perpetrators of forced penetration were men. In total, this suggests forced penetration is a dramatically under-recognized form of sexual victimization affecting heterosexual men that is mostly perpetrated by heterosexual women.

Indeed, nearly $5 \%$ of college men perpetrate rape (Anderson, Silver, Ciampaglia, Vitale, \& Delahanty, 2019 ) and that is considered a public health crisis (Freire-Vargas, 2018). Yet rarely is forced penetration against men discussed and women's perpetration is often considered extraordinarily rare (Stemple, Flores, \& Meyer, 2017; Weiss, 2010). This underscores how problematic it is that currently, there are no standardized measures of sexual victimization that assess forced penetration. There are some measures that may capture these experiences - such as the Revised Conflict Tactics Scales and the Post-Refusal Sexual Persistence Scale - Victimization - as the structure of these questionnaires are broad enough to include this behavior. Indeed, it is likely that the broad definitions of sex and gender neutral wording that records higher prevalence rates on these questionnaires when compared to measures such as the SESSFV. However, these questionnaires are so broad as to be non-specific about any individual sexual victimization experience and thus, unable to provide estimates for specific experiences.

Consistent with hypotheses and prior research such as Weare (20I8a), forced penetration was most often perpetrated by women - this was demonstrated both by men's accounts of their victimization and direct reports of perpetration from women $(\mathrm{H} 2)$. This convergence of evidence further supports Weare (2018a)'s findings. Also consistent with hypotheses was that forced penetration was more often 
perpetrated by romantic partners. This suggests that forced penetration may be most common in established heterosexual relationships. Notably, and somewhat surprisingly to us, our results indicate that forced penetration is not exclusive to heterosexual women but we consider these findings more tentative given missing data and lack of comparative literature.

We examined patterns of missing data as a proxy for participant understanding of the items. Missing data patterns were consistent with prevalence findings - that is women, who are the least likely to experienced forced penetration victimization were the most likely to skip items (RQ3). However, our assessment of missing data suggests that the items tested in this study may cause confusion for some participants. Regarding the forced penetration victimization items approximately $6-20 \%$ of participants skipped these items; otherwise missing data was extremely low in Samples I and 2. Thus, these items stood out in being more likely to be skipped by participants. Notably in Study 2, missing data was even higher, nearly $50 \%$ for the perpetration items. However, this is confounded with a change in sample - Sample 2 consisted of MTurk workers and MTurk workers tend to produce lower quality data. This, combined with Buday \& Peterson's (20I5) findings regarding $40 \%$ of women producing victimization data on a perpetration questionnaire suggests it may be difficult to accurately capture forced penetration perpetration data.

Validity

Yet, our findings regarding the validity of the perpetration items suggested strong evidence of validity, as these items were strongly correlated with other perpetration items $(\mathrm{HIb})$. The forced penetration victimization items also demonstrated evidence of validity with correlations mostly in the hypothesized range $(\mathrm{HIa})$. Alternatively, the patterns of missing data and validity correlations could also suggest a lack of validity of the forced penetration items tested in this study. It may be that missing data was high because the items were difficult to understand and that the victimization items were less strongly correlated because of spurious responding. However, this seems unlikely given that the items tested in this study were very similar to those used by Weare (2018a) which were then validated qualitatively. With this less conservative interpretation of the validity correlations, future research is warranted to investigate ways to decrease missing data and provide additional evidence of validity.

Implications for Future Research, Clinical Practice, and Policy

Our findings suggest further research into forced penetration is imperative. Of particular importance is research into how to best assess forced penetration. Although the items used in this study demonstrate initial evidence of validity, further evidence is needed as is evidence of reliability. Given Weare (20I8b)'s findings regarding the traumatic impact of forced penetration, further research is also needed on the health impact of these experiences. Our findings are in contrast to the CDC report as we suggest that the prevalence rate of women's forced penetration victimization is above zero - further research is needed to confirm that women do experience this. This also suggests that legal advocacy and policy change is important as forced penetration does affect a significant number of people, especially men. Our data also suggest women's perpetration of forced penetration is fairly common and in need of specific preventative efforts as well as further study. We strongly recommend that clinical service providers inquire about forced penetration and that health and legal professionals advocate for policy change to better recognize this form of sexual victimization (Weiss, 2010). This lack of data and corresponding clinical work and cultural narrative contributes to the stigma and shame men may feel regarding their experiences of victimization (Aakvaag, Thoresen, Wentzel-Larsen, Dyb, Roysamb, \& Olff, 2016; Sable et al., 2006).

\section{Limitations}

A significant number, $(7.4-13.1 \%)$ of college men reported experiencing forced penetration in this study (RQI), higher than previous research. In the NISVS study, almost 5\% of men reported forced penetration. The most common form of forced penetration victimization reported by men was vaginal, whereas this was attempted anal for women. In these studies, forced penetration accounted for $33.8-$ $58.7 \%$ of cases of men's sexual victimization. Additionally, 9.2-13.04\% of men's cases of sexual victimization were uniquely accounted for by forced penetration, i.e., they would have been labeled as unaffected had we not assessed forced penetration, suggesting that not measuring this type of victimization may dramatically underestimate the extent of men's sexual victimization. Notably, given the prevalence of vaginal forced penetration in our samples, this also suggests women's perpetration is being vastly underestimated. 


\section{Conclusions}

This is one of the first studies to assess the validity of items designed to assess forced penetration. Our findings suggest forced penetration accounts for approximately one third of cases of men's sexual victimization and is most often perpetrated by women. All of the unique cases of forced penetration identified were of heterosexual men underscoring the unique gender and sexual identity dynamics at work in forced penetration cases. In sum, our findings suggest that both men's sexual victimization and women's sexual perpetration is woefully under-researched and in need of further scrutiny to reduce the public health burden of sexual violence.

\section{Declaration of Conflicting Interests}

The author(s) declared no potential conflicts of interest with respect to the research, authorship, and/or publication of this article.

\section{Funding}

Dr. Anderson and Ms. Goodman's work on this paper was supported by grant \#5K01AA026643-02 from the National Institutes on Alcohol Abuse and Alcoholism. Views presented in this paper do not represent those of the granting agency. Participants were paid on MTurk via a small grant awarded to Dr. Anderson from the Applied Psychology Center at Kent State University.

\section{ORCID iD}

RaeAnn E. Anderson (i) https://orcid.org/0000-0001-9938-0717

\section{References}

Aakvaag, H. F., Thoresen, S., Wentzel-Larsen, T., Dyb, G., Røysamb, E., \& Olff, M. (2016). Broken and guilty since it happened: A population study of trauma-related shame and guilt after violence and sexual abuse. Journal of Affective Disorders, 204, 16-23. https://doi.org/10.1016/j.jad.2016.06.004

Anderson, R. E., \& Delahanty, D. L. (2019). Discrepant responding across measures of college students' sexual victimization experiences: Conceptual replication and extension. The Journal of Sex Research, 1-12.

Anderson, R. E., Cahill, S. P., \& Delahanty, D. L. (2017). Initial Evidence for the Reliability and Validity of the Sexual Experience Survey-Short Form Perpetration (SES-SFP) in College Men. Journal of Aggression, Maltreatment \& Trauma. https://doi.org/10.1080/10926771.2017.1330296

Anderson, R. E., Cahill, S. P., \& Delahanty, D. L. (2018a). The Psychometric Properties of the Sexual Experiences SurveyShort Form Victimization (SES-SFV) and Characteristics of Sexual Victimization Experiences in College Men. Psychology of Men and Masculinity, 19(1), 25-34. https://doi.org/10.1037/men0000073

Anderson, R. E., Cahill, S. P., \& Delahanty, D. L. (2018b). Discordance Between the Sexual Experiences Surveys-Short Forms and the Revised Conflict Tactics Scales in College Men. Psychology of Violence. https://doi.org/10.1037/vio0000199

Anderson, R. E., Silver, K. E., Ciampaglia, A. M., Vitale, A. M., \& Delahanty, D. L. (2019). The Frequency of Sexual Perpetration in College Men: A Systematic Review of Reported Prevalence Rates From 2000 to 2017. Trauma, Violence, \& Abuse, 152483801986061. https://doi.org/10.1177/1524838019860619

Anthony, E. R., \& Cook, S. L. (2012). Assessing the impact of gender-neutral language on dis- closure of sexual violence. Psychology of Violence, 2(3), 297-307. https://doi.org/10.1037/ a0028562

Basile, K. C., Smith, S., Breiding, M. J., Black, M. C., \& Mahendra, R. (2014). Sexual Violence Surveillance: Uniform Definitions and Recommended Data Elements, Version 2.0. Retrieved from https://www.cdc.gov/violenceprevention/pdf/sv_surveillance_definitionsl-2009-a.pdf

Black, M. C., Basile, K. C., Breiding, M. J., Smith, S. G., Walters, M. L., Merrick, M. T., Chen, J., \& Stevens, M. R., (2011). The National Intimate Partner and Sexual Violence Survey (NISVS): 2010 Summary Report. Atlanta, GA: National Center for Injury Prevention and Control, Centers for Disease Control and Prevention.

Buday, S. K., \& Peterson, Z. D. (2015). Men's and Women's Interpretation and Endorsement of Items Measuring SelfReported Heterosexual Aggression. Journal of Sex Research, 52(9), 1042-1053. https://doi.org/10.1080/00224499.2014.967373

Canan, S. N., Jozkowski, K. N., Wiersma-Mosley, J. D., Bradley, M., \& Blunt-Vinti, H. (2019). Differences in Lesbian, Bisexual, and Heterosexual Women's Experiences of Sexual Assault and Rape in a National U.S. Sample. Journal of Interpersonal Violence, 088626051986372. https://doi.org/10.1177/0886260519863725 
Choudhary, E., Coben, J., \& Bossarte, R. M. (2009). Adverse Health Outcomes, Perpetrator Characteristics, and Sexual Violence Victimization Among U.S. Adult Males. Journal of Interpersonal Violence, 25(8), 15231541. https://doi.org/10.1177/0886260509346063

Davies, M. (2002). Male sexual assault victims: a selective review of the literature and implications for support services. Aggression \& Violent Behavior, 7(3), 203. https://doi.org/10.1016/S1359-1789(00)00043-4

Diamantopoulos, A., Riefler, P., \& Roth, K. P. (2008). Advancing formative measurement models. Journal of Business Research, 61(12), 1203-1218. https://doi.org/10.1016/j. jbusres.2008.01.009

Dworkin, E. R., Menon, S. V., Bystrynski, J., \& Allen, N. E. (2017). Sexual assault victimization and psychopathology: A review and meta-analysis. Clinical psychology review, 56, 65-81. https://doi.org/10.1016/j.cpr.2017.06.002

Fisher, N. L., \& Pina, A. (2013). An overview of the literature on female-perpetrated adult male sexual victimization. Aggression and Violent Behavior, 18(1), 54-61. https://doi.org/10.1016/j.avb.2012.10.001

Freire-Vargas, L. (2018, January 1). Violence as a public health crisis. AMA Journal of Ethics, Vol. 20, pp. $25-28$. https://doi.org/10.1001/journalofethics.2018.20.1.fred1-1801

Hipp, T. N., \& Cook, S. L. (2017). Rape and sexual assault on campus, in diverse populations, and in the spotlight. In C. M. Renzetti, J. L. Edleson, \& R. K. Bergen (Eds.), Sourcebook on Violence Against Women (3rd ed., pp. 85-101). Sage Publications.

Johnson, S. M., Murphy, M. J., \& Gidycz, C. A. (2017). Reliability and validity of the Sexual Experiences Survey - Short Forms victimization and perpetration. Violence and Victims, 32(1), 78-92. https://doi.org/10.1891/0886-6708.VV-D-1500110

French, B. H., Tilghman, J. D., \& Malebranche, D. A. (2015). Sexual coercion context and psychosocial correlates among diverse males. Psychology of Men \& Masculinity, 16(1), 42. https://doi.org/10.1037/a0035915

Koss, M. P. (1993). Rape: Scope, impact, interventions, and public policy responses. American Psychologist, 48(10), 1062 1069. https://doi.org/10.1037/0003-066X.48.10.1062

Koss, M. P., \& Gidycz, C. A. (1985). Sexual Experiences Survey: Reliability and validity. Journal of Consulting and Clinical Psychology, 53(3), 422-423. https://doi.org/10.1037/0022-006X.53.3.422

Koss, M. P., Abbey, A., Campbell, R., Cook, S., Norris, J., Testa, M., ... White, J. (2007). Revising the SES: A Collaborative process to improve assessment of sexual aggression and victimization. Psychology of Women Quarterly, 31(4), 357-370. https://doi.org/10.1111/j.1471-6402.2007.00385.x

Ouimette, P. C., Shaw, J., Drozd, J. F., \& Leader, J. (2000). Consistency of reports of rape behaviors among nonincarcerated men. Psychology of Men \& Masculinity, 1(2), 133-139.

Peterson, Z. D., Voller, E. K., Polusny, M. A., \& Murdoch, M. (2010). Prevalence and consequences of adult sexual assault of men: review of empirical findings and state of the literature. Clinical Psychology Review, 31(1), 1-24. https://doi.org/10.1016/j.cpr.2010.08.006

Ross, R. R., \& Allgeier, E. R. (1996). Behind the pencil/paper measurement of sexual coercion: Interview-based clarification of men's interpretations of Sexual Experiences Survey items. Journal of Applied Social Psychology, 26, 1587-1616. https://doi.org/10.1111/j.1559-1816.1996.tb00087.x

Sable, M. R., Danis, F., Mauzy, D. L., \& Gallagher, S. K. (2006). Barriers to reporting sexual assault for women and men: perspectives of college students. Journal of American College Health, 55(3), 157-162. https://doi.org/10.3200/JACH.55.3.157-162

Stemple, L., \& Meyer, I. H. (2014). The sexual victimization of men in America: New data challenge old assumptions. American Journal of Public Health, 104(6), e19-e26.

Stemple, L., Flores, A., \& Meyer, I. H. (2017). Sexual victimization perpetrated by women: Federal data reveal surprising prevalence. Aggression and Violent Behavior, Vol. 34, pp. 302-311. https://doi.org/10.1016/j.avb.2016.09.007

Struckman-Johnson, C., Struckman-Johnson, D., Anderson, P. B., Struckman-Johnson, C., Struckman-Johnson, D., \& Anderson, P. B. (2003). Tactics of sexual coercion: when men and women won't take no for an answer. Journal of Sex Research, 40(1), 76-86. https://doi.org/10.1080/00224490309552168

Turchik, J. A. (2012). Sexual victimization among male college students: Assault severity, sexual functioning, and health risk behaviors. Psychology of Men \& Masculinity, 13(3), 243. https://doi.org/10.1037/a0024605

Weare, S. (2018a). From Coercion to Physical Force: Aggressive Strategies Used by Women Against Men in "Forced-toPenetrate" Cases in the UK. Archives of Sexual Behavior, 47(8), 2191-2205. https://doi.org/10.1007/s10508-018-12325

Weare, S. (2018b). "I Feel Permanently Traumatized By It": Physical and Emotional Impacts Reported by Men Forced to Penetrate Women in the United Kingdom. Journal of Interpersonal Violence, 088626051882081. https://doi.org/10.1177/0886260518820815

Wegner, R., \& Davis, K. C. (2017). How Men's Sexual Assault Victimization Experiences Differ Based on Their Sexual History. Journal of Interpersonal Violence, 088626051770337. https://doi.org/10.1177/0886260517703374 
Weiss, K. G. (2010). Male sexual victimization: Examining men's experiences of rape and sexual assault. Men and Masculinities, 12(3), 275-298. https://doi.org/10.1177/1097184X08322632

\section{Author Biographies}

RaeAnn E. Anderson (PhD, University of Wisconsin-Milwaukee, 2015) is currently an assis- tant professor in Clinical Psychology at the University of North Dakota. She completed her postdoctoral training at Kent State University and her undergraduate degree at University of Kansas. Her research interests are understanding basic behavioral processes in sexual victimiza- tion and sexual perpetration in order to inform sexual assault risk reduction and prevention programs, respectively.

Erica L. Goodman (MA, University of North Dakota, 20I7) has a master's degree in clinical psychology and is currently on doctoral internship at Northwestern University. Her thesis used structural equation modeling to assess comorbid indicators of non-suicidal self-injury and eat- ing disorder psychopathology. Her dissertation is investigating facets of loss-of-control eating in a non-clinical sample. Erica continues to do clinical work and research surrounding issues related to eating pathology and, recently, sexual violence perpetration and victimization.

Sidney S. Thimm (Undergraduate, University of North Dakota) is currently pursuing her undergraduate degree in Psychology and is in her second year at University of North Dakota. She is planning on going to graduate school to get her doctorate in either forensic or clinical psychology. She has been working in Dr. Anderson's lab for over a year researching sexual violence victimization and perpetration. 
Table I

Comparison of Number of Cases of Forced Penetration Victimization by Gender with Percentage of Cases by Type of Forced Penetration Noted for Men

\begin{tabular}{|c|c|c|c|c|}
\hline & $\begin{array}{l}\text { Completed } \\
\text { Vaginal }\end{array}$ & $\begin{array}{l}\text { At- } \\
\text { tempted } \\
\text { Anal } \\
\end{array}$ & $\begin{array}{l}\text { Completed } \\
\text { Anal }\end{array}$ & $\begin{array}{l}\text { Any Forced Penetra- } \\
\text { tion }\end{array}$ \\
\hline \multicolumn{5}{|l|}{ Sample I, N = 673} \\
\hline Men, $n=298$ & 19 (100.0\%) & I (10.0\%) & $4(100.0 \%)$ & 22 (7.04\% of men) \\
\hline Women, $n=367$ & 0 & 9 & 0 & 9 (2.45\% of women) \\
\hline Total cases & 19 & 10 & 4 & 31 (4.45\% of sample) \\
\hline \multicolumn{5}{|l|}{ Sample 2, N = 783} \\
\hline Men, $n=206$ & $18(62.06 \%)$ & $12(20 \%)$ & $12(50.0 \%)$ & 27 (13.11\% of men) \\
\hline Women, $n=567$ & 11 & 48 & 12 & 55 (9.70\% of women) \\
\hline Total cases & 29 & 60 & 24 & 82 ( $10.47 \%$ of sample) \\
\hline
\end{tabular}

Table 2

Correlation between Forced Penetration (FP) Victimization Items and Standard SES-SFV Items

\begin{tabular}{|c|c|c|c|c|c|c|}
\hline \multicolumn{4}{|c|}{ Sample $1, N=672$} & \multicolumn{3}{|c|}{$\frac{\text { Sample } 2, N=}{792}$} \\
\hline & FP I & FP 2 & FP 3 & FP I & $\overline{\text { FP } 2}$ & FP 3 \\
\hline \multicolumn{7}{|l|}{ FP items } \\
\hline$\overline{\text { vaginal }(1)}$ & 一 & .330 & .128 & 一 & .302 & .344 \\
\hline anal (2) & 一 & - & .286 & - & - & .555 \\
\hline att. anal (3) & - & 一 & - & - & - & - \\
\hline$\underline{\text { SES-SFV items }}$ & - & 一 & 一 & 一 & - & 一 \\
\hline $1-$ touching & .226 & .228 & .391 & .342 & .316 & .479 \\
\hline 2 - oral & .260 & .303 & .461 & .452 & .384 & .517 \\
\hline 3 - vaginal & .269 & .362 & .503 & .455 & .298 & .540 \\
\hline $4-$ anal & .272 & .438 & .565 & .234 & .633 & .621 \\
\hline 5 - att. oral & .118 & .236 & .378 & .479 & .534 & .443 \\
\hline 6 - att. vaginal & .097 & .042 & .486 & .206 & .221 & .418 \\
\hline 7 - att. anal & .152 & .223 & .663 & .306 & .433 & .481 \\
\hline
\end{tabular}

Note. FP = forced penetration, att $=$ attempted. SES-SFV = Sexual Experiences Survey - Short Form Victimization. All correlations statistically significant, $p<.00$ I, except the underlined values which were not significantly related. 
Table 3

Characteristics of the Perpetrators of Forced Penetration According to Victims/Survivors

\begin{tabular}{|c|c|c|c|c|c|}
\hline Gender of victim & $\begin{array}{l}\text { Perp. Gen- } \\
\text { der - \% } \\
\text { Women }\end{array}$ & $\begin{array}{l}\text { Romantic } \\
\text { Partner }\end{array}$ & Acquaintance & Stranger & Multiple Perp. \\
\hline \multicolumn{6}{|l|}{ Study I } \\
\hline Men FP & $72.7 \% * \wedge$ & $68.2 \% *$ & $40.9 \%$ & $18.2 \%$ & $13.6 \%$ \\
\hline Women FP & $0 \%$ & $87.5 \%$ & $75 \%$ & $37.5 \%$ & $25 \%$ \\
\hline Other Cases - Men & $47.5 \%$ & $45.8 \%$ & $33.9 \%$ & $13.6 \%$ & $10.2 \%$ \\
\hline Other Cases - Women & $1.6 \%$ & $36.5 \%$ & $45.0 \%$ & $9.0 \%$ & $8.5 \%$ \\
\hline \multicolumn{6}{|l|}{ Study 2} \\
\hline Men FP & $37 \% \wedge$ & $29.6 \% * \wedge$ & $29.6 \%$ & $18.5 \%$ & $25.9 \%$ \\
\hline Women FP & $87.3 \% *$ & $63.6 \%$ & $47.3 \%$ & $20 \%$ & $16.4 \%$ \\
\hline Other Cases - Men & $33.3 \%$ & $16.7 \%$ & - & - & - \\
\hline Other Cases - Women & $3.2 \%$ & $48.6 \%$ & $34.4 \%$ & $11.7 \%$ & $5.3 \%$ \\
\hline
\end{tabular}

Note. Perp. $=$ perpetration. FP $=$ forced penetration, $*=$ indicates significant comparison to Other Victimization Cases of the same gender. ${ }^{\wedge}=$ indicates significant comparison to Women who experienced FP victimization

Table 4

Correlations between Forced Penetration (FP) Perpetration Items and Standard SES-SFP Items, N = 283

FP I FP 2 FP 3 FP4

\begin{tabular}{l|llll}
\hline FP items & & & & \\
vaginal (I) & - & .862 & .798 & .848 \\
anal (2) & - & - & .866 & .958 \\
att. vaginal (3) & - & - & - & .804 \\
att. anal (4) & - & - & - & - \\
SES-SFP items & - & - & - & - \\
\hline I- touching & .639 & .756 & .713 & .764 \\
$2-$ oral & $.76 I$ & .885 & .836 & .819 \\
$3-$ vaginal & .503 & .667 & .552 & .632 \\
$4-$ anal & .862 & .985 & .866 & .948 \\
$5-$ att. oral & .827 & .864 & .934 & .840 \\
$6-$ att. vaginal & .933 & .874 & .706 & .832 \\
$7-$ att. anal & .793 & .889 & .810 & .873
\end{tabular}

Note. FP = forced penetration, SES-SFP = Sexual Experiences Survey - Short Form Perpetration. All correlations statistically significant, $p<.001$. 